\title{
Cell Surface Changes Accompanying the Neural Differentiation of an Embryonal Carcinoma Cell Line
}

\author{
Joel M. Levine and Patricia Flynn \\ Department of Neurobiology and Behavior, State University of New York at Stony Brook, Stony Brook, New York \\ 11794
}

\begin{abstract}
The murine embryonal carcinoma cell line P19S1801A1 develops into neuronlike cells after treatment with retinoic acid (Edwards and McBurney, 1983). We have analyzed the expression of cell surface carbohydrate antigens and intracellular cytoskeletal antigens in differentiating $01 \mathrm{Al}$ cells in order to identify the cell types present in the cultures and to characterize the differentiation process. Undifferentiated $01 \mathrm{~A} 1$ cells express the SSEA-1 antigen, $\mathrm{GD}_{3}$ ganglioside, and the D1.1 ganglioside antigen, carbohydrate markers that are found on early embryonic cells and neuroepithelial germinal cells in vivo. The cells also bind tetanus toxin, cholera toxin, and monoclonal antibody A2B5, probes that bind to gangliosides found on the surfaces of neurons and immature astrocytes in vivo and in vitro. They contain vimentin-type intermediate filament antigens but have no detectable neurofilament or glial filament protein antigens. After aggregation of the cells in medium containing retinoic acid followed by growth in a serum-free chemically defined medium, over $80 \%$ of the cells differentiate into neurons as determined by immunofluorescent labeling with antibodies against neurofilament protein antigens. The differentiated cells no longer express either the embryonic or neuroepithelial carbohydrate antigens, but they continue to express the cell surface markers characteristic of neurons. These changes in the expression of cell surface antigens are accompanied by changes in ganglioside metabolism, including a shift towards the synthesis of more complex gangliosides. Thus, the retinoic acid-induced changes in $01 \mathrm{A1}$ cells in vitro resemble the in vivo development of neurons. This establishes the $\mathrm{O} 1 \mathrm{A1}$ cell line as a relevant model system for studies of the molecular basis of neuronal differentiation and development.
\end{abstract}

The differentiation of embryonic ectoderm to neuroectoderm and the subsequent proliferation of neurons and glial cells has been described, yet the cellular and molccular mcchanisms underlying this sequence of events remain largely unknown. The multiplicity of cell types found in both the CNS and PNS and their discoordinate development in vivo severely limit our ability to analyze the mechanisms responsible for neuronal and glial differentiation. Furthermore, it is often difficult to obtain the large amounts of material needed for biochemical and molecular studies from early mammalian embryos.

Embryonal carcinoma cell lines are established from germ line tumors and preimplantation embryos and have been used as model systems for studying the early cvents of mammalian

\footnotetext{
Received Mar. 18, 1986; revised June 6, 1986; accepted June 12, 1986.

We wish to thank Dr. M. McBurney for providing us with the O1Al cell line, Drs. D. Dahl, D. Paulin, and D. Solter for their gifts of antibodies, and Drs. S. Halegoua and $\mathrm{J}$. White for their comments on this manuscript. This work was supported by a grant from the NIH.

Correspondence should be addressed to Dr. Joel Levine at the above address. Copyright (C) 1986 Society for Neuroscience $0270-6474 / 86 / 113374-11 \$ 02.00 / 0$
}

development and differentiation (Martin, 1980). In response to exogenous factors such as dibutyryl AMP, retinoic acid (RA), or dimethyl sulfoxide (DMSO), these pluripotent cell lines differcntiate into a variety of cell types, including neurons (Edwards and McBurney, 1983; Jones-Villeneuve et al., 1982; Thompson et al., 1984; Wartiovaara et al., 1984). One cell line, P19S1801A1 (termed O1A1) responds to RA in a dose-dependent manner: Low concentrations of RA or exposure to DMSO leads to the development of muscle cells, whereas a higher concentration of RA causes the cells to differentiate into neurons and astrocytes (Edwards and McBurney, 1983). The ability to transform a homogeneous population of cells with the properties of pluripotent embryonic cells into neurons may provide a useful model system for the analysis of neuronal development. Ilowever, if such a model system is to be relevant, the in vitro differentiation should resemble the in vivo development of neurons and glial cells.

We show here that O1Al cells can be induced by RA treatment to differentiate into neuronlike cells at high yield and with minimal contamination by other cell types. This RA-induced differentiation is accompanied by multiple changes in the repertoire of carbohydrate antigens present on the cell surface, and these changes are similar to those reported to occur during the in vivo differentiation of ncuroepithclial cells, the germinal cells of the CNS. The O1Al cell line therefore may provide a valuable model system for the analysis of the molecular events underlying neuronal differentiation.

\section{Materials and Methods}

\section{Cell culture.}

The P19S1801A1 cell line was obtained from Dr. M. McBurney and maintained in culture in Dulbecco's modified Eagle's medium (DMEM; GIBCO) supplemented with $10 \%$ fetal calf serum (FCS; K-C Biologicals). For neuronal differentiation, the cells were plated into petri dishes (Falcon) at a density of $10^{5} / \mathrm{ml}$ in DMEM containing $10 \% \mathrm{FCS}$ and $5 \times$ $10^{-7} \mathrm{M}$ RA (Sigma). After $48 \mathrm{hr}$ in culture, during which time the cells formed large floating aggregates, the aggregates were harvested, washed in DMEM containing $20 \mathrm{mM}$ HEPES and replated into petri dishes in DMEM containing $10 \%$ FCS. Following an additional $48 \mathrm{hr}$ of culture as aggregates, the cells were harvested, washed, and treated with $0.25 \%$ trypsin (Worthington) in calcium-free PBS for $10 \mathrm{~min}$ at $37^{\circ} \mathrm{C}$. The aggregates were then washed, mechanically dissociated with a flamenarrowed Pasteur pipette and plated into poly-L-lysine-coated tissue culture dishes (Falcon). The plating medium was the chemically defined N2 medium of Bottenstein and Sato (1978) supplemented with $5 \mu \mathrm{g} /$ $\mathrm{ml}$ human fibronectin (Boehringer Mannheim). Cells were fed at $3 \mathrm{~d}$ intervals with unsupplemented N2 medium. In some experiments, the growth of non-neuronal cells was suppressed by treating the cultures with $10^{-5} \mathrm{M}$ cytosine arabinoside (Sigma).

\section{Immunofluorescent methods and antibodies}

The immunofluorescent staining of living cells in monolayer culture was performed as described previously (Levine et al., 1984). The following 


\begin{tabular}{lll}
\hline Table 1. Antibody and toxin probes & \\
Probe & Cell surface ligand & Distribution \\
\hline Monoclonal D1.1 & $O$-acetylated ganglioside & Neuroepithelial cells \\
Monoclonal R24 & $\mathrm{GD}_{3}$ ganglioside & Neuroepithelial cells \\
Monoclonal SSEA-1 & Gal $\beta$ l $\rightarrow$ 4GlcNac-R & EC cell lines, mouse neural tube \\
& $\downarrow$ & \\
Monoclonal A2B5 & Polysialo-gangliosides & Neurons, glial cells \\
Cholera toxin & $\mathrm{GM}_{1}$ ganglioside & Neurons \\
Tetanus toxin & $\mathrm{GD}_{\mathrm{bb}}$ and GT gangliosides & Neurons, immature astrocytes \\
\hline
\end{tabular}

antibody and toxin probes were used to detect cell surface carbohydrate antigens: monoclonal antibody D1.1 (Levine et al., 1984), monoclonal antibody A2B5 (Eisenbarth et al., 1979) obtained from ATCC, monoclonal antibody SSEA-1 (Solter and Knowles, 1978) obtained from D. Solter, monoclonal antibody R24 (Pukel et al., 1982; Cambridge Research Labs.), fluorescein-labeled cholera toxin B subunit (List Biological Laboratories, Inc.), tetanus toxin (Calbiochem), and horse antitetanus toxin (Connaught Labs LTD). The binding of the mouse monoclonal antibodies was detected with fluorescein- or rhodamine-conjugated goat anti-mouse (TAGO). Fluorescein-labeled goat anti-horse was purchased from Cappel. For the detection of intracellular antigens, the cells were fixed with either $95 \%$ ethanol or formaldehyde as described by Hynes and Destree (1978). A monoclonal human anti-vimentin antibody (Dellagi et al., 1982) and a monoclonal mouse anti-neurofilament antibody were gifts from Dr. D. Paulin, Pasteur Institute. Rabbit antiglial fibrillary acidic protein (GFAP) was purchased from DAKO, and a polyclonal rabbit anti-neurofilament antibody was a gift from Dr. D. Dahl. Rabbit antibodies against melanocyte-stimulating hormone (MSH), which cross-react with neurofilament antigens (Drager et al., 1983), were purchased from Immunonuclear. Fluorescein- and rhodamine-conjugated goat anti-rabbit antibodies were prepared in our laboratory, and rhodamine-conjugated goat anti-human was purchased from TAGO. The cells were examined with a Leitz Dialux 22 microscope equipped for epifluorescent and phase illumination and photographed on Kodak Tri-X film using a Leitz Vario-orthomat II camera.

\section{Ganglioside preparation and analysis}

Gangliosides were prepared from cells using the method of Suzuki as described by Ledeen and Yu (1982). The upper-phase lipids were desalted on Sep-Pak C18 cartridges (Waters Associates) according to Williams and McCluer (1980). Gangliosides were metabolically labeled by growing the cells for 48-72 hr in medium supplemented with $5 \mu \mathrm{Ci} / \mathrm{ml}$ ${ }^{3} \mathrm{H}$-galactose (ICN; specific activity, $25 \mathrm{Ci} / \mathrm{mmol}$ ) and $5 \mu \mathrm{Ci} / \mathrm{ml}{ }^{3} \mathrm{H}$ glucosamine (ICN; specific activity, $40 \mathrm{Ci} / \mathrm{mmol}$ ). Thin-layer chromatography (TLC) was performed as described (Levine et al., 1984) using HPTLC silica gel 60 plates purchased from EM Merck. The radioactive gangliosides were detected by fluorography on preflashed film after spraying the plates with Enhance (New England Nuclear). Radioimmune binding to gangliosides separated on aluminum-backed TLC plates was performed as described previously (Levine et al., 1984). The binding of monoclonal anti-D1.1 was detected using ${ }^{125}$ I-labeled $\mathrm{Fab}_{2}$ fragments of goat anti-mouse IgM (TAGO), and for monoclonal R24, ${ }^{125}$ I-labeled protein A was used. Cells were enzymatically labeled with ${ }^{125} I$ as described previously (Stallcup et al., 1983). Immune precipitation and SDS-PAGE and autoradiography were performed as described (Stallcup et al., 1983).

\section{Results}

Table 1 lists the antibodies and bacterial toxins used as probes for cell surface carbohydrate antigens in this study. By analyzing the expression of well-characterized carbohydrate antigens, we compared the cell surface phenotype of differentiating O1A1 cells with that of developing neurons and glial cells. SSEA-1, a carbohydrate antigen associated with glycoproteins and glycolipids (Gooi et al., 1981; Ozawa et al., 1985), is found on many human and murine EC cell lines (Solter and Knowles, 1978) and has been localized to the mouse neural tube in vivo (Fox et al., 1981). Monoclonal antibodies D1.1 and R24 bind to ganglioside antigens that are found specifically on the surfaces of proliferating neuroepithelial cells (Levine et al., 1984; Goldman et al., 1984). Monoclonal antibody A2B5 (Eisenbarth et al., 1979) recognizes gangliosides that are found on the surfaces of neurons and astrocytes in vitro (Schnitzer and Schachner, 1982). Tetanus toxin and cholera toxin bind to gangliosides on the surfaces of neurons, although this specificity is not absolute (Raff et al., 1983; Willinger and Schachner, 1980). In addition to analyzing the cell surface phenotype of differentiating O1Al cells, we used a panel of antibodies against intermediate filament protein antigens to identify the cell types present in the differentiating cultures.

\section{Phenotype of the undifferentiated cells}

Undifferentiated O1A 1 cells grew rapidly in monolayer cultures in the presence of $10 \%$ FCS. Most of the cells were phase dark and polygonal in shape, although some phase bright, round cells were also present in the cultures (Fig. 1A). Some of the cells had short, thick cytoplasmic extrusions and smaller microspikes, but at no time were cells with neuritic processes observed in the cultures. As has been reported for other murine and human EC cell lines (Solter and Knowles, 1978), greater than $90 \%$ of the cells bound monoclonal anti-SSEA-1 (Fig. 2, $G$ and $H$ ). Approximately $90 \%$ of the cells bound the surface markers for proliferating neuroepithelial cells, monoclonal antibodies D1.1 and R24 (Fig. 2, $A-D$ ). Thirty percent of the cells bound monoclonal antibody A2B5 (Fig. 2, $E$ and $F$ ). Labeling with the A2B5 antibody revealed the presence of numerous microspike-like processes. In addition, greater than $90 \%$ of the cells bound tetanus toxin and cholera toxin (Fig. 3). The cells were unstained when labeled with antibodies against neurofilament protein antigens or against GFAP (data not shown), but all the cells were labeled with antibodies against vimentin, an intermediate filament marker of mesodermal cells and cells of the developing neural tube-Figure 4, $A$ and $B$ (Bignami et al., 1982; Cochard and Paulin, 1984; Lazarides, 1980). Thus, the pluripotent EC cell line O1Al has a cell surface and intracellular phenotype that is similar in several regards to that of primitive neuroectodermal cells.

\section{Phenotype of the differentiated cells}

Following aggregation in the presence of $5 \times 10^{-7} \mathrm{M}$ RA and 2 $\mathrm{d}$ of culture without $\mathrm{RA}$, the aggregates were dissociated into single cells and plated onto poly-L-lysine-coated tissue culture dishes. The cells attached to the substrate rapidly and within 2-3 hr began to elaborate neuritic processes. Over the next 2$3 \mathrm{~d}$ of culture, greater than $80 \%$ of the cells developed rounded cell bodies with 1 or more processes (Fig. 1C). In contrast, when the RA-treated cells were dissociated and plated in medium containing $10 \%$ FCS, many fewer cells developed a neuronal appearance (Fig. $1 D$ ). The serum-containing cultures were characterized by a rapid overgrowth of non-neuronal cells, some of 

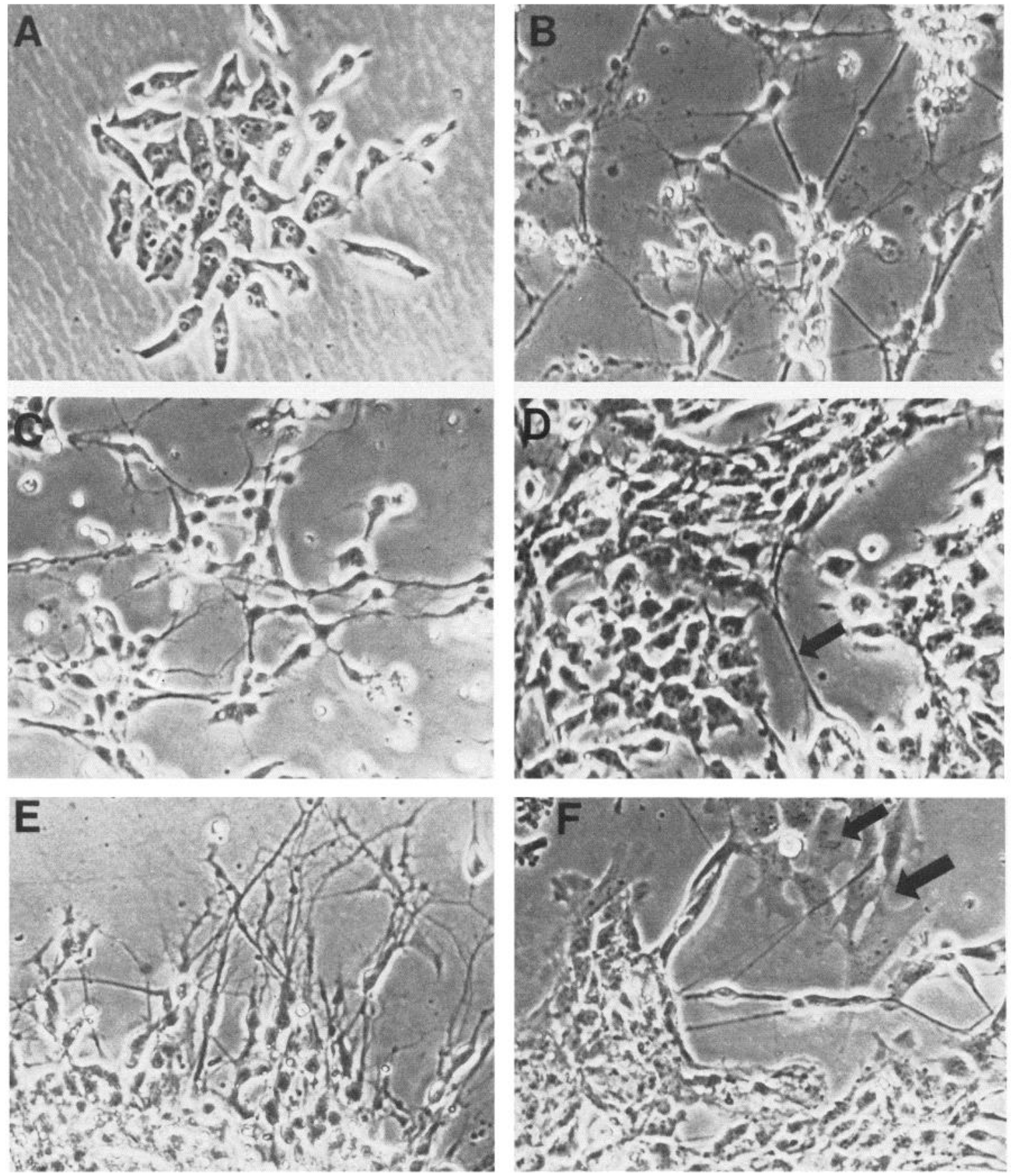

Figure 1. Morphological appearance of O1A1 cells. $A$, Undifferentiated O1A1 cells. $B$, O1A1 cells dissociated from aggregates previously treated with $5 \times 10^{-7} \mathrm{M} \mathrm{RA}$ and grown in N2 medium for $11 \mathrm{~d}$. $C$, Differentiated cells plated as dissociated cells and grown for $2 \mathrm{~d}$ in N2 medium. $D$, Differentiated cells placed as dissociated cells and grown for $2 \mathrm{~d}$ in serum-containing medium; the arrow points to a neuritic process. $E$, Differentiated cells plated as aggregates and grown for $2 \mathrm{~d}$ in $\mathrm{N} 2$ medium. $F$, Differentiated cells plated as aggregates and grown in serum-containing medium for $2 \mathrm{~d}$; the arrows denote a cluster of flat fibroblastic cells. $\times 450$.

which had a flattened fibroblastic appearance. The effects of defined medium on the development of neuronal characteristics were also evident when the RA-treated cells were plated as aggregates. Figure $1 E$ shows the periphery of an aggregate grown in N2 medium with extensive neurite outgrowth. Aggregates grown in serum-containing medium were more heterogeneous, containing a mixture of cell types, only a few of which had neuritic processes $($ Fig. $1 F$ ). The neuronal-appearing cells could be maintained in N2 medium for up to $20 \mathrm{~d}$, although most experiments were performed using cells that had been grown in defined medium for between 5-12 d (Fig. 1B).
We used a panel of antibodies against intermediate protein antigens to monitor the differentiation of the cultures and to identify the cell types present. Figure 4 shows that after 3-5 d of differentiation, the process-bearing cells were labeled with polyclonal and monoclonal antibodies that recognize neurofilament protein antigens. The expression of vimentin-like intermediate filament antigens was significantly reduced in the differentiated cultures, with immunofluorescent staining restricted to the flat fibroblastic cells (Fig. 4, $I-K$ ). During the first 3-5 d of culture in defined medium, there were some cells in the cultures with shorter processes that were positive for both neuro- 

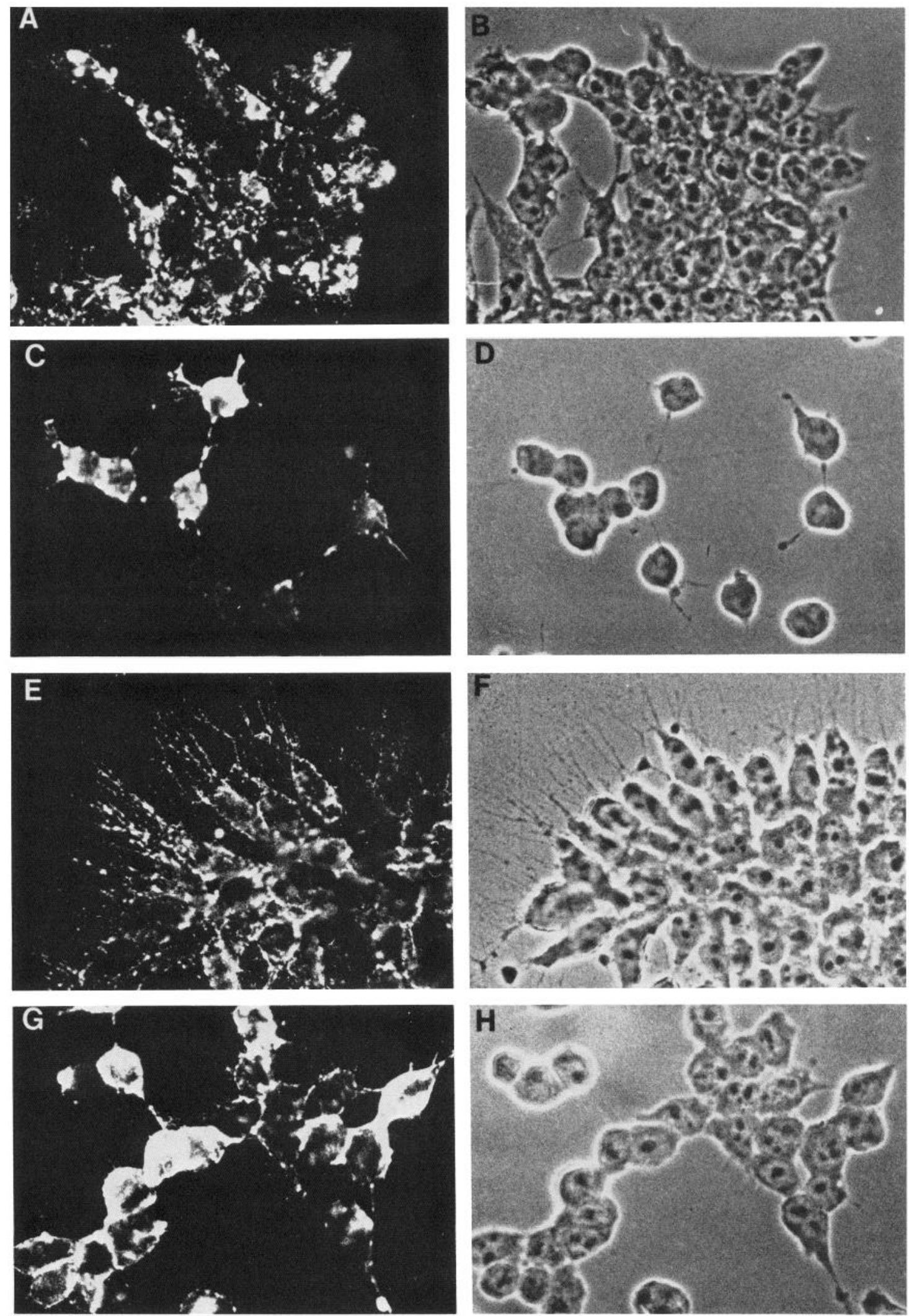

Figure 2. Cell surface antigens of undifferentiated O1Al cells. Cells were immunofluorescently labeled as described under Materials and Methods. $A$, Anti-D1.1 staining. $B$, Phase, C-R24 staining. $D$, Phase. $E$, A2B5 staining. $F$, Phase. $G$, SSEA-1 staining. $H$, Phase. $\times 395$. 

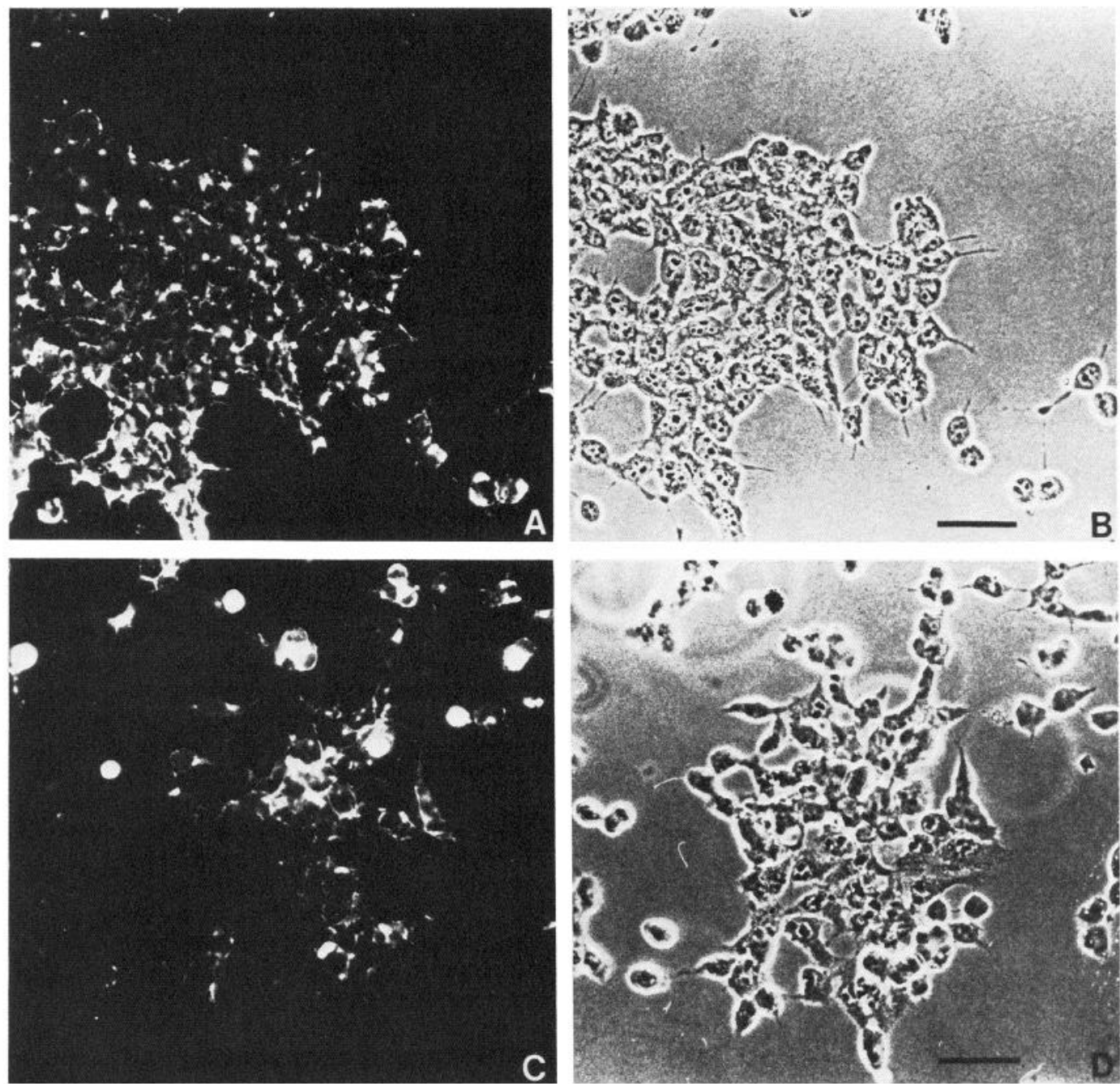

Figure 3. Labeling of undifferentiated O1A1 cells with bacterial toxins. $A$, Tetanus toxin. $B$, Phase. $C$, Cholera toxin. $D$, Phase. Bar, $50 \mu \mathrm{m}$.

filament antigens and vimentin (data not shown). Less than $0.1 \%$ of the cells were labeled with rabbit antibodies against GFAP (Fig. 4, $G$ and $H$ ). Many of the GFAP-positive cells tended to appear in areas of the cultures containing clumps of cells that had not been dissociated completely. This suggests that cell-cell contact may be necessary for the astrocytic differentiation of O1A1 cells, although other alternatives such as local depletion of the medium have not been ruled out.

The expression of cell surface carbohydrate antigens by the differentiated cells is shown in Figure 5 and Table 2. After $5 \mathrm{~d}$ in monolayer culture in defined medium, less than $5 \%$ of the cells bound monoclonal antibodies D1.1, R24, and SSEA-1. The few labeled cells in the cultures may represent either O1A1 cells that did not respond to RA (Campione-Piccardo et al., 1985) or cells that were delayed in their neuronal differentiation. Monoclonal antibody D1.1 labeled a small number of cells with processes in cultures that had been grown as monolayers for 24 $\mathrm{hr}$. When the cells were labeled after $3 \mathrm{~d}$ in monolayer culture, no staining with the anti-D1.1 antibody was seen. Twenty to $40 \%$ of the cells bound monoclonal antibody A2B5. Figure 5 , $A$ and $B$, shows a bipolar cell labeled with the A2B5 antibody that has extremely long processes. All of the process-bearing cells in the cultures were labeled with both tetanus toxin and cholera toxin (Fig. 5, $C-F$ ). The labeling with cholera toxin was heaviest on the cell bodies, whereas tetanus toxin labeled cell bodies and processes. The flat cells without processes were not labeled with tetanus toxin. These data demonstrate that the RAinduced neuronal differentiation of O1Al cells is accompanied by a reduction in the expression of cell surface carbohydrate antigens characteristic of early embryonic cells and neuroepithelial germinal cells.

To determine whether the loss of the neuroectodermal cell

Table 2. Percent of labeled O1A1 cells

\begin{tabular}{lcccccc} 
& & \multicolumn{2}{c}{ Aggregates } & & \multicolumn{2}{c}{ Differentiated cells } \\
\cline { 3 - 4 } Probe & Undiff. & $3 \mathrm{~d}$ & $5 \mathrm{~d}$ & & $3 \mathrm{~d}$ & $5 \mathrm{~d}$ \\
\hline D1.1 & $>90$ & 50 & $<10$ & & $<5$ & $<5$ \\
R24 & $>90$ & 90 & $<10$ & & $<5$ & $<5$ \\
A2B5 & $20-30$ & 80 & 50 & & 20 & $20-40$ \\
SSEA-1 & $>90$ & 90 & $30-50$ & & $<5$ & $<5$ \\
CT & $>90$ & $>90$ & 80 & & $>90$ & $>90$ \\
TT & $>90$ & $>90$ & $>90$ & & $>90$ & $>90$
\end{tabular}

At the indicated stages of the differentiation process, cells were immunofluorescently labeled with the probes as described under Materials and Methods and the number of labeled cells counted. Between 200-400 cells were counted for each determination; however, because of cell clumping, the numbers given represent approximations. Undiff., undifferentiated cells; CT, cholera toxin; TT, tetanus toxin. 

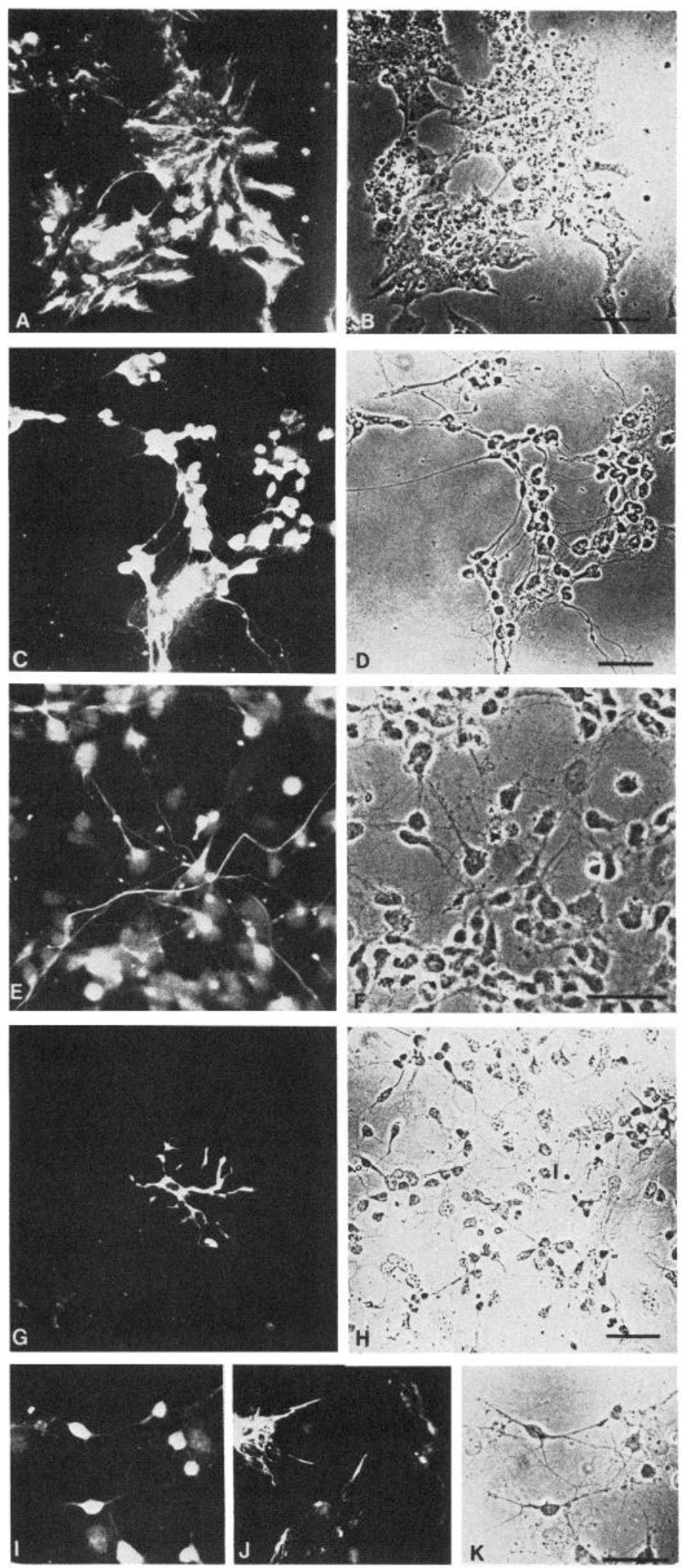

Figure 4. Expression of intermediate filament protein antigens by $\mathrm{OlAl}$ cells. $A$, undifferentiated cells, anti-vimentin label. $B$, Phase. $C$, Six day differentiated cells, rabbit anti-neurofilament label, with the plane of focus on the cell bodies. $D$, Phase. $E$, Four day differentiated cells, rabbit anti- $\alpha$-MSH label, with the plane of focus on the neuritic processes. $F$, Phase. $G$, Three day differentiated cells, rabbit anti-GFAP label. $H$, Phase. $I$, Eleven day differentiated cells, anti-neurofilament label. $J$, Same field, monoclonal anti-vimentin label. $K$, Phase. Bar, $50 \mu \mathrm{m}$. surface markers described above was a consequence of the RAinduced differentiation into neuron-like cells or whether this loss was a general effect of differentiation, we aggregated cells in the presence of $1 \%$ DMSO and grew the dissociated cells in either $\mathrm{N} 2$ medium or in DMEM containing 10\% FCS. The resulting cultures contained a mixture of cell types including fibroblastic cells and myoblast-like cells (Edwards et al., 1983). Approximately $80 \%$ of the mesodermally differentiated cells were labeled with the anti-vimentin antibody, but no stained cells were seen after labeling with either anti-neurofilament or anti-GFAP antibodies (data not shown). Long-term cell survival in defined medium was poor, but after $4 \mathrm{~d}$ of monolayer culture, $30 \%$ of the cells were labeled with anti-D1.1, 20\% with R24, $72 \%$ with SSEA-1, $8 \%$ with A2B5, $25 \%$ with tetanus toxin and $8 \%$ with cholera toxin. The mesodermal cells induced by DMSO treatment survived better in medium containing FCS and after $4 \mathrm{~d}$ of culture, $70 \%$ of the cells were labeled with anti-D1.1, $37 \%$ of the cells were labeled with R $24,76 \%$ with SSEA- 1 , and $31 \%$ with A2B5. Twelve percent of the cells bound tetanus toxin, and $48 \%$ were labeled with fluorescent cholera toxin. These data show that the almost complete loss of the neuroectodermal and early embryonic surface markers is a consequence of aggregation in RA and does not occur after aggregation in the presence of agents capable of stimulating mesodermal differentiation. The induction of the expression of neurofilament or glial filament protein antigens occurs under conditions that cause the O1A1 cells to develop into neuron-like cells but not under conditions that foster the development of muscle cells.

\section{Cell surface changes during aggregation}

We analyzed cell surface changes that occur during the aggregation process by dissociating 3 and $5 \mathrm{~d}$ aggregates, allowing the cells to attach to poly-L-lysine-coated surfaces and labeling the cells with the monoclonal antibodies listed in Table 1 . Table 2 shows that after $3 \mathrm{~d}$ of aggregation in RA, there is an increase in the percentage of cells labeled with monoclonal antibody A2B5 and a reduction in the number of anti-D1.1-positive cells with little change in the cell surface expression of GD3 ganglioside, which is the antigen recognized by antibody R24 (Pukel et al., 1982). Following 2 additional d of culture as aggregates, D1.1, R24, and A2B5 labeling is significantly reduced. Thus, it appears that several days of aggregate culture are necessary for the cell surface expression of the metabolic changes initiated by treatment with RA.

\section{Biochemical correlates of neuronal differentiation}

The cell surface and intracellular changes described above were all deduced from antibody labeling studies. Changes in ganglioside metabolism accompanying neuronal differentiation were also analyzed by metabolic labeling studies and immunostaining of TLC chromatograms. Figure $6 \mathrm{~A}$ is a fluorogram of metabolically labeled gangliosides prepared from undifferentiated and neurally differentiated O1A1 cells. Although all of the bands seen in Figure $6 A$ have not been identified, the neuronally differentiated cells synthesized increased amounts of more complex (more slowly migrating and more highly sialyated) gangliosides. A densitometric analysis of these metabolic changes is given in Figure $6 \mathrm{~B}$. Labeling of bands numbered 1, 2, 5, 6, 7,8 , and 9 was reduced 6 - to 30 -fold in the differentiated cells when compared to the undifferentiated stock cells, and bands numbered $3,4,10,11,12$, and 13 were increased 4 - to 6 -fold. A similar shift in ganglioside metabolism towards the synthesis of more complex gangliosides has been observed in several studies of ganglioside metabolism in developing brain (Rosner, 1982; Yavin and Yavin, 1979).

To identify the molecules responsible for antibody binding to the cell surface, we labeled the surfaces of both undifferen- 

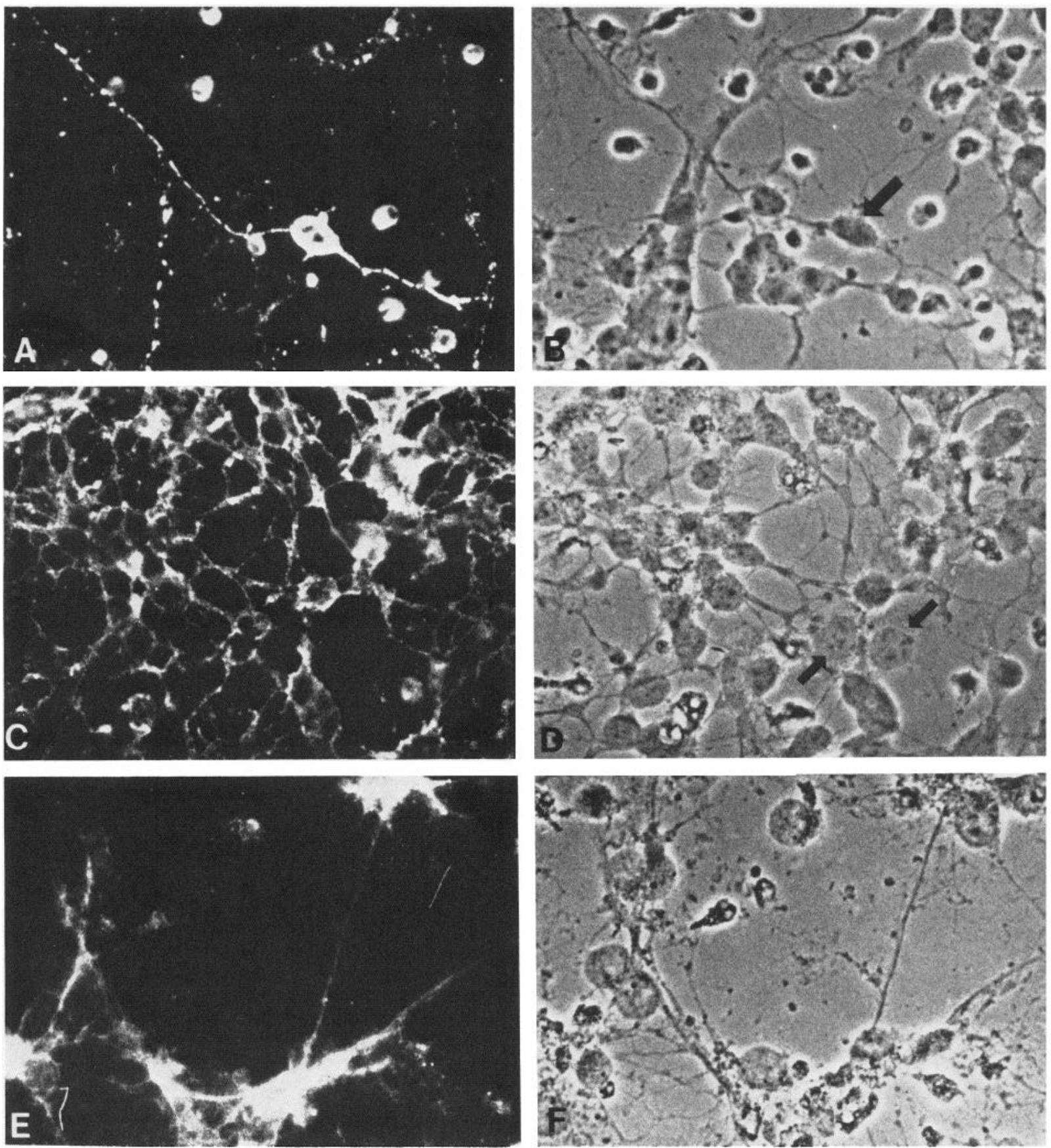

Figure 5. Cell surface labeling of neurally differentiated cells. A, Four day differentiated cells, A2B5 label. B, Phase; the arrow points to the A2B5 labeled cell. $C$. Four day differentiated cells, tetanus toxin label. $D$, Phase; the arrows indicate 2 flat cells that are not labeled with tetanus toxin. $E$, Ten day differentiated cells, cholera toxin label. $F$, Phase. $\times 440$.

tiated and differentiated O1A1 cells with ${ }^{125} \mathrm{I}$ as described under Materials and Methods, lysed the cells in nonionic detergents, and performed immune precipitations on aliquots of the lysates. Neither anti-D1.1, R24, A2B5, nor tetanus toxin was able to precipitate labeled polypeptides from the lysates; however, antibody SSEA-1 precipitated a high-molecular-weight smear only from the undifferentiated cells (data not shown). Immunostaining of gangliosides separated on TLC chromatograms was used to analyze the expression of the D1.1 and R24 antigens during the differentiation process. Figure $7 \mathrm{~A}$ shows that the undifferentiated O1A1 cells synthesize 1 molecular species of ganglio- side that binds anti-D1.1. This ganglioside comigrates with the D1.1 antigen present on B49 cells (the cell line against which the antibody was raised); thus, it is likely that anti-D1.1 is recognizing an $O$-acetylated form of $\mathrm{GD}_{3}$ present on $\mathrm{O} 1 \mathrm{~A} 1$ cells (Cheresh et al., 1984b). The decline in the amount of immunostainable D1.1 antigen seen in Figure $7 A$ is consistent with the data in Table 2 . Immunostaining of TLC chromatograms with R24 (Fig. $7 B$ ) shows that the relative amounts of $\mathrm{GD}_{3}$ ganglioside in the differentiated cells are significantly reduced. As stated above, there was negligible immunofluorescent staining of the differentiated cultures with R24. The small amount 


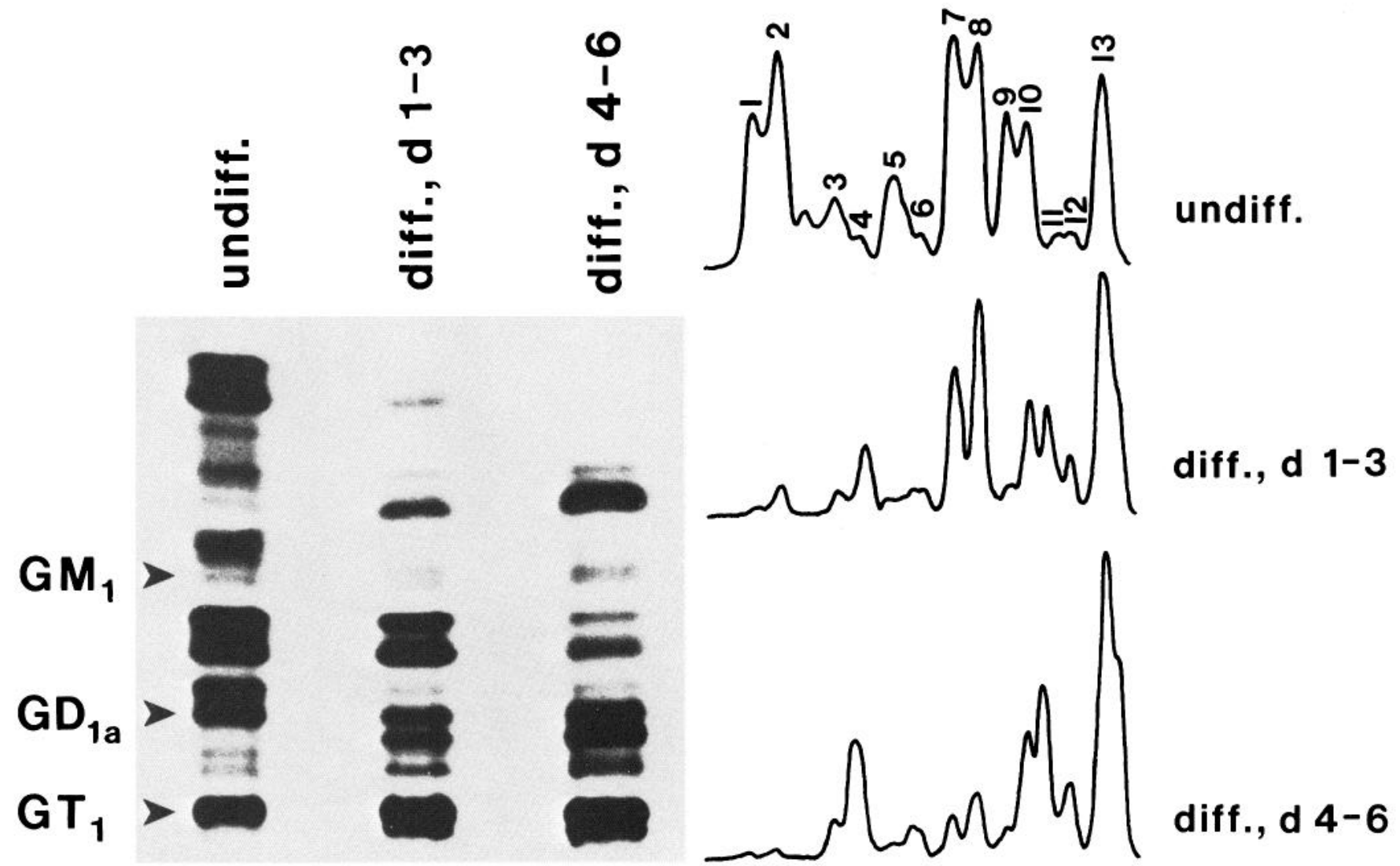

Figure 6. Ganglioside biosynthesis by differentiating O1A1 cells. A, Fluorogram of metabolically labeled gangliosides prepared from undifferentiated cells (undiff.), differentiated cells labeled on days 1-3 of monolayer culture (diff., $d$ 1-3) and differentiated cells labeled on days 4-6 of monolayer culture (diff., $d 4-6$ ). ${ }^{3} \mathrm{H}$-ganglioside, $15,000 \mathrm{cpm}$, was loaded in each lane. The mobilities of standard gangliosides, visualized with $\mathrm{I}_{2}$ vapor, are shown at left. $B$, Densitometric analysis of the fluorogram shown in $A$. The numbers refer to bands mentioned in the text, and the top of each lane is to the left.

of $\mathrm{GD}_{3}$ detected by immunostaining of gangliosides from the neuronal cells may represent intracellular pools of $\mathrm{GD}_{3}$ that can act as precursor in the biosynthesis of more complex gangliosides (Fishman and Brady, 1976).

\section{Discussion}

The following conclusions can be drawn from the data presented. (1) The O1A1 cell line responds to RA by differentiating predominantly into neuron-like cells; (2) differentiated O1A1 cells, but not the undifferentiated cells, display neurofilamentlike immunoreactivity, a reliable marker for the neuronal phenotype; (3) the neuronal differentiation of O1A1 cells is accompanied by a loss at the cell surface of carbohydrate antigens characteristic of early embryonic and neuroepithelial germinal cells; (4) RA-induced neuronal differentiation is accompanied by major changes in ganglioside metabolism and expression at the cell surface. These conclusions suggest that the O1A1 cell line provides a valuable model system for the in vitro analysis of neuronal differentiation.

McBurney and colleagues (Edwards et al., 1983; Jones-Villeneuve et al., 1982) first demonstrated that the OlAl cell line and its parental line P19 can differentiate into neuron-like cells after treatment with RA. Under their conditions, however, the resulting cultures contained both neurons and glial cells. Two minor changes in the methods developed by McBurney resulted in a more homogeneous population of neuron-like cells in our cultures. First, we dissociated the aggregates into a single-cell suspension and then plated the cells onto surfaces made adhesive by coating with poly-L-lysine. The use of poly-L-lysine resulted in an increased plating efficiency. Dissociation of the aggregates disrupted the cell-cell interactions that may play a role in the development of astrocytes in the cultures. In control experiments, intact aggregates were plated onto either coated or uncoated tissue culture dishes. The aggregates attached to the substrate rather slowly, and the resulting cultures contained neurons, astrocytes and unidentified flat cells. Second, we grew the dissociated cells in the chemically defined N2 medium. This medium was originally designed to promote the growth and morphological differentiation of neuroblastoma cells (Bottenstein and Sato, 1978); subsequent studies have shown that neurons can survive and acquire differentiated functions when grown in serum-free, chemically defined media (Ahmed et al., 1984; Skaper et al., 1979). Defined medium also promotes neurite outgrowth and the acquisition of neuronal properties in cultures of premigratory chick neural crest (Ziller et al., 1983) and other embryonal carcinoma cell lines (Darmon et al., 1982). We have noted that undifferentiated O1A1 cells divide slowly and survive poorly when grown directly in defined medium (unpublished 


\section{A B}

Figure 7. Analysis of the expression of the D1.1 and R24 antigens during the neural differentiation of O1A1 cells. TLC and immunostaining were done as described under Materials and Methods. A, TLC chromatogram probed with anti-D1.1 and ${ }^{125}$ I-goat anti-mouse $\mathrm{Fab}_{2}$ antibodies: $B 49,1 \mu \mathrm{g}$ of gangliosides prepared from B49 cells was spotted and used as a standard to show the mobility of the D1.1 antigen; undiff., undifferentiated $\mathrm{O} 1 \mathrm{~A} 1$ cells; $3 d$ agg., aggregates that had been treated with RA for $48 \mathrm{hr}$; $5 d$ agg., aggregates that had been grown for an additional $48 \mathrm{hr}$ after RA treatment; $5 d$ diff., differentiated cells in monolayer culture for $5 \mathrm{~d}$. For the O1Al samples, gangliosides equivalent to 15 $\mathrm{mg}$ tissue wet weight were loaded in each lane. $B, \mathrm{R} 24$ and ${ }^{125} \mathrm{I}$-protein A immunostaining of gangliosides. Lanes were loaded as described in $A$ except that a ganglioside extract of embryonic day 17 rat brain (E17 brain) was used as a standard to show the mobility of $\mathrm{GD}_{3}$ ganglioside.
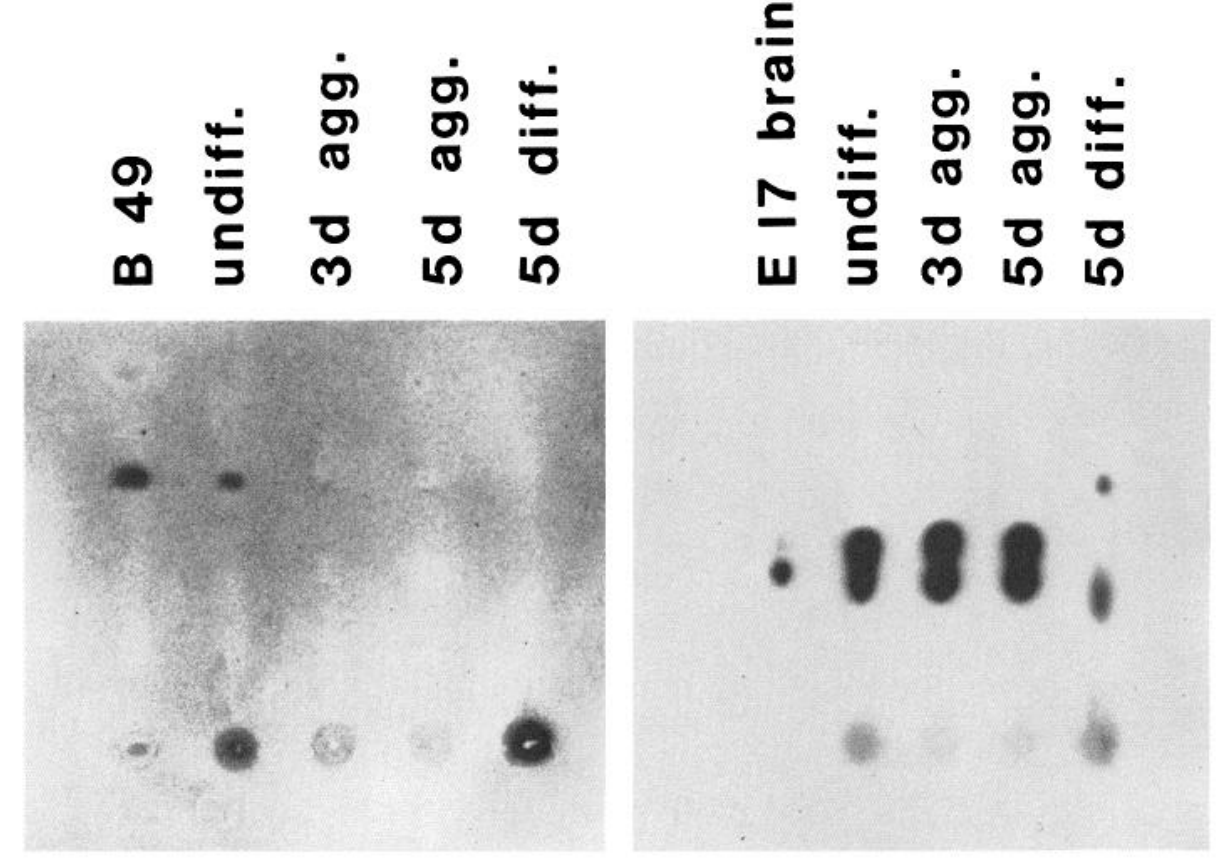

observations). Therefore, any O1A1 stem cells that do not respond to RA do not survive and proliferate under our conditions. The mechanism of action of RA is unknown; however, the identification of a cytosolic RA-binding protein has led to the suggestion that retinoids may affect gene expression in a manner analogous to that of steroid hormones (Jetten and Jetten, 1979). It is likely that aggregation in RA primes the cells in some manner for neuronal differentiation and that defined medium allows for the maximum expression of neuronal characteristics.

In this study, we used a panel of antibodies and bacterial toxins that recognize cell surface carbohydrate antigens and intermediate filament proteins. Five classes of intermediate filaments have been recognized (Lazarides, 1980), among them neurofilament proteins and GFAP are cell-type-specific markers for neurons and astrocytes, respectively. Vimentin, on the other hand, is widespread and found in many cell types, including neuroepithelial germinal cells (Bignami et al., 1981; Cochard and Paulin, 1984). The presence of neurofilament-like immunoreactivity, detected with several different antibodies, demonstrates that the cells in our cultures are neuronal in nature. The neurofilament-positive differentiated O1A1 cells develop from vimentin-positive EC stem cells in a manner analagous to the development of neurofilament-positive neurons from vimentin-positive germinal cells in the neural tube (Bignami et al., 1982; Cochard and Paulin, 1984). Thus, the sequential expression of intermediate filament protein antigens known to occur in vivo is recapitulated in the cell culture system described here.

The D1.1 ganglioside antigen and $\mathrm{GD}_{3}$, the ganglioside recognized by monoclonal antibody R24, are markers for dividing neuroepithelial cells (Goldman et al., 1984; Levine et al., 1984). In vivo and in vitro immunofluorescent studies have demonstrated that, as a germinal cell stops dividing and its daughter cells differentiate into neurons, the differentiated cells no longer express the $\mathrm{D} 1.1$ or $\mathrm{GD}_{3}$ ganglioside on their surfaces. A parallel loss of antibody binding to the cell surface of differentiating
O1A1 cells is demonstrated in this study, suggesting that at least some of the mechanisms of differentiation that act in vivo operate during the in vitro differentiation described here.

Monoclonal antibody D1.1 can recognize several gangliosides of brain and sensory neurons, all of which are likely to contain $O$-acetyl sialic acid (Levine et al., 1986). The major antigen recognized by anti-D1.1 in the developing cerebellum and in the B49 cell line appears to be related structurally to $\mathrm{GD}_{3}$, with the only difference being the presence of 9-O-acetyl sialic acid (Cheresh et al., 1984a). The loss of D1.1 from the cell surface precedes the loss of $\mathrm{GD}_{3}$ (Fig. 7), suggesting that 1 of the initial steps in neuronal differentiation may be a reduction in the activity of a sialic acid $O$-acetylase, which can convert $\mathrm{GD}_{3}$ into a form recognized by antibody D1.1 (Cheresh et al., 1984b). Using O1A1 cells as a model system, it may be possible to elucidate the mechanisms responsible for the antigenic changes, as well as the other changes in ganglioside metabolism evident from our studies.

In addition to the ganglioside changes discussed above, the O1A1 cells constitutively express other ganglioside markers for neurons. The nature of the antigens recognized by monoclonal antibody A2B5 is controversial (Fredman et al., 1984; Kasai and $\mathrm{Yu}, 1983$ ), and we have not attempted to identify the molecule(s) responsible for antibody binding to O1A1 cells. The biosynthesis of $\mathrm{GM}_{1}$, which is the membrane receptor for cholera toxin (Cuatrecasas, 1973; Fishman et al., 1977), is essentially unchanged in the differentiated cells compared to the undifferentiated cells (band 6, Fig. 6B). On the other hand, the neuronal cells appear to make considerably more $\mathrm{GT}_{1}$ and $\mathrm{GD}_{1 \mathrm{~b}}$ (bands 13 and 11 and 12 , respectively; Fig. $6 B$ ), which act as receptors for tetanus toxin (Dimpfel et al., 1977). Although the presence of these neuronal markers on the undifferentiated cells could be taken to indicate a commitment to a neuronal lineage, the fact that lower concentrations of RA or DMSO can induce a mesodermal phenotype in the cells makes this conclusion unwarranted. The mesodermal cells induced by aggregation in DMSO have fewer binding sites for tetanus toxin and cholera toxin, 
demonstrating that the quantitative expression of the ganglioside receptors for these toxins can be modified in differentiating OlAl cells.

Cell lines have provided useful model systems for the analysis of neuronal functions. For example, studies of the PC12 cell line have revealed details of the mechanism of action of NGF (McTigue et al., 1985), and neuroblastoma lines have been used to study neurotransmitter receptor function (Sharma et al., 1975). The O1A1 cell line has several properties that suggest it, too, may comprise a useful model system. The cells are pluripotent and capable of developing into neurons, astroglia, or muscle cells (Edwards and McBurney, 1983; Edwards et al., 1983). O1 Al cells are defective in thymidine kinase (McBurney et al., 1982), making them suitable for gene-transfer experiments. We have recently shown that O1A1 cells synthesize and process pro-opiomelanocortin-like material to $\beta$-endorphins (Fuller et al., 1985). Most important, the RA-induced differentiation resembles the in vivo development of neurons with regard to cell surface carbohydrate antigen expression and intermediate filament protein expression. Thus, they can be used to study the events that occur during the terminal differentiation of neuroectoderm, something that is not currently possible using cells isolated from mammalian embryos.

\section{References}

Ahmed, Z., P. S. Walker and R. E. Fellows (1984) Properties of neurons from dissociated fetal rat brain in serum-free culture. J. Neurosci. 3: 2448-2462.

Bignami, A., T. Raju, and D. Dahl (1982) Localization of vimentin, the nonspecific intermediate filament protein, in embryonal glia and in early differentiating neurons. Dev. Biol. 91: 286-295.

Bottenstein, J. E., and G. H. Sato (1978) Growth of a rat neuroblastoma cell line in serum-free supplemented medium. Proc. Natl. Acad. Sci. USA $76: 514-517$

Campione-Piccardo, J., J. Craig, J. J. Sun, and M. W. McBurney (1985) Commitment in a murine embryonal carcinoma cell line during differentiation induced by retinoic acid. Exp. Cell Res. 156: 544-552.

Cheresh, D. A., R. A. Reisfeld, and A. P. Varki. (1984a) O-Acetylation of disialoganglioside $\mathrm{GD}_{3}$ by human melanoma cells creates a unique antigenic determinant. Science 225: 844-846.

Cheresh, D. A., A. P. Varki, N. M. Varki, W. B. Stallcup, J. Levine, and R. A. Reisfeld (1984b) A monoclonal antibody recognizes an $O$-acylated sialic acid in a human melanoma-associated ganglioside. J. Biol. Chem. 259: 7453-7459.

Cochard, P., and D. Paulin (1984) Initial expression of neurofilaments and vimentin in the central and peripheral nervous system of the mouse embryo in vivo. J. Neurosci. 4: 2080-2094.

Cuatrecasas, $P$. (1973) Gangliosides and membrane receptors for cholera toxin. Biochemistry 12: 3558-3566.

Darmon, M., W. B. Stallcup, and Q. J. Pittman (1982) Induction of neural differentiation by serum-deprivation in cultures of the embryonal carcinoma cell line 1003. Exp. Cell Res. 138: 73-78.

Dellagi, K., J. C. Brouet, J. Perreau, and D. Paulin (1982) Human monoclonal IgM with autoantibody activity against intermediate filaments. Proc. Natl. Acad. Sci. USA. 79: 446-450.

Dimpfel, W., R. Huang, and E. Habermann (1977) Gangliosides in nervous tissue cultures and binding of ${ }^{125}$ I-labeled tetanus toxin, a neuronal marker. J. Neurochem. 29: 329-334.

Drager, U. C., D. L. Edwards, and J. Kleinschmidt (1983) Neurofilaments contain $\alpha$-melanocyte-stimulating hormone ( $\alpha$-MSH)-like immunoreactivity. Proc. Natl. Acad. Sci. USA 80: 6408-6412.

Edwards, M. K., and M. W. McBurney (1983) The concentration of retinoic acid determines the differentiated cell types formed by a teratocarcinoma cell line. Dev. Biol. 98: 187-191.

Edwards, M. K., J. F. Harris, and M. W. McBurney (1983) Induced muscle differentiation in an embryonal carcinoma cell line. Mol. Cell. Biol. 3: 2280-2286.

Eisenbarth, G. S., F. S. Walsh, and M. Niremberg (1979) Monoclonal antibody to a plasma membrane antigen of neurons. Proc. Natl. Acad. Sci. USA 76: 4913-4917.

Fishman, P. H., and R. O. Brady (1976) Biosynthesis and function of gangliosides. Science 194: 906-915.
Fishman, P. H., J. Moss, and J. C. Osborne (1977) Interaction of choleragen with the oligosaccharide of ganglioside GM1: Evidence for multiple binding sites. Biochemistry $17: 711-716$.

Fox, N., I. Damjanov, A. Martinez-Hernandez, B. B. Knowles, and D. Solter (1981) Immunohistochemical localization of the early embryonic antigen (SSEA-1) in postimplantation mouse embryos and fetal and adult tissues. Dev. Biol. 83: 391-398.

Fredman, P., J. L. Magnani, M. Nirenberger, and V. Ginsburg (1984) Monoclonal antibody $\mathrm{A} 2 \mathrm{~B} 5$ reacts with many gangliosides in neuronal tissue. Arch. Biochem. Biophys. 223: 661-666.

Fuller, J. A., J. M. Levine, and J. F. McKelvy (1985) Biosynthesis of POMC-derived peptides in a differentiated embryonal carcinoma cell line. Soc. Neurosci. Abstr. 11: 566.

Goldman, J. E., M. Hirano, R. K. Yu, and T. N. Seyfried (1984) $\mathrm{GD}_{3}$ ganglioside is a glycolipid characteristic of immature neuroectodermal cells. J. Neuroimmunol. 7: 179-192.

Gooi, H. C., T. Feize, A. Kapadia, B. B. Knowles, D. Solter, and M. J. Evans (1981) Stage-specific embryonic antigen involves 1-3 fucosylated type 2 blood chains. Nature 292: 156-158.

Hynes, R. O., and A. T. Destree (1978) $10 \mathrm{~nm}$ filaments in normal and transformed cells. Cell 13: 151-163.

Jetten, A. M., and M. E. R. Jetten (1979) Possible role of retinoic acid binding protein in retinoid stimulations of embryonal carcinoma cell differentiation. Nature 278: 180-182.

Jones-Villeneuve, E. M. V., M. W. McBurney, K. A. Rogers, and V. I. Kalnins (1982) Retinoic acid induces embryonal carcinoma cells to differentiate into neurons and glial cells. J. Cell Biol. 94: 253-262.

Kasai, N., and R. K. Yu (1983) The monoclonal antibody A2B5 is specific to ganglioside $\mathrm{GQ}_{1 \mathrm{c}}$. Brain Res. 277: 155-158.

Lazarides, E. (1980) Intermediate filaments as mechanical integrators of cellular space. Nature 283: 249-256.

Ledeen, R. W., and R. K. Yu (1982) Gangliosides: Structure, isolation and analysis. Methods Enzymol. 83: 139-191.

Levine, J. M., L. Beasley, and W. Stallcup (1984) The D1.1 antigen: A cell surface marker for germinal cells of the central nervous system. J. Neurosci. 4: 820-831.

Levine, J. M., L. Beasley, and W. B. Stallcup (1986) Localization of a neurectoderm-associated cell surface antigen in the developing and adult rat. Dev. Brain Res. 27: 211-222.

Martin, G. R. (1980) Teratocarcinomas and mammalian embryogenesis. Science 209: 768-776.

McBurney, M. W., E. M. V. Jones-Villeneuve, M. K. S. Edwards, and P. J. Anderson (1982) Control of muscle and neuronal differentiation in a cultured embryonal carcinoma cell line. Nature 299: 165167.

McTigue, M., J. Cremins, and S. Halegoua (1985) Nerve growth factor and other agents mediate phosphorylation and activation of tyrosine hydroxylase. J. Biol. Chem. 260: 9047-9056.

Ozawa, M., J. Muramatsu, and D. Solter (1985) SSEA-1, a stagespecific embryonic antigen is carried by the glycoprotein bound large carbohydrate in embryonal carcinoma cells. Cell Diff. 16: 169-173.

Pukel, C. S., K. O. Lloyd, L. R. Travassos, W. G. Dippold, H. F. Oettgen, and L. J. Old (1982) GD3, a prominent ganglioside of human melanoma. J. Exp. Med. 155: 1133-1147.

Raff, M. C., R. H. Miller, and M. Noble (1983) A glial progenitor cell that develops in vitro into an astrocyte or an oligodendrocyte depending on culture media. Nature 303: 390-396.

Rosner, H. (1982) Ganglioside changes in the chicken optic lobes as biochemical indicators of brain development and maturation. Brain Res. 236: 49-61.

Schnitzer, J., and M. Schachner (1982) Cell type specificity of a neural cell surface antigen recognized by the monoclonal antibody A2B5. Cell Tissue Res. 224: 625-636.

Sharma, S. K., W. A. Klee, and M. Nirenberg (1975) Dual regulation of adenylate cyclase accounts for narcotic dependence and tolerance. Proc. Natl. Acad. Sci. USA 72: 3092-3096.

Skaper, S. D., R. Adler, and S. Varon (1979) A procedure for purifying neuron-like cells in cultures from central nervous tissue with a defined medium. Dev. Neurosci. 2: 233-237.

Solter, D., and B. B. Knowles (1978) Monoclonal antibody defining a stage-specific mouse embryonic antigen (SSEA-1). Proc. Natl. Acad. Sci. USA 75: 5565-5569.

Stallcup, W. B., L. S. Arner, and J. M. Levine (1983) An antiserum against the PCl 2 cell line defines cell surface antigens specific for neurons and Schwann cells. J. Neurosci. 3: 53-68. 
Thompson, S., P. L. Stern, M. Webb, F. S. Walsh, W. Engstrom, E. P. Evans, W.-K. Shi, B. Hopkins, and C. F. Graham (1984) Cloned human teratoma cells differentiate into neuron-like cells and other cell types in retinoic acid. J. Cell. Sci. 72: 37-64.

Wartiovaara, J., P. Liesi, and L. Rechardt (1984) Neuronal differentiation in F9 embryonal carcinoma cells. Cell Diff. 15: 125-128.

Williams, M. A., and R. H. McCluer (1980) The use of Sep-Pak C 18 cartridges during the isolation of gangliosides. J. Neurochem. 35:266269.

Willinger, M., and M. Schachner (1980) GM1 ganglioside as a marker for neuronal differentiation in mouse cerebellum. Dev. Biol. 74: 101117.

Yavin, E., and Z. Yavin (1979) Ganglioside profiles during neural tissue development. Acquisition in the prenatal rat brain and cerebral cell cultures. Dev. Neurosci. 2: 25-37.

Ziller, C., E. Dupin, P. Brazeau, D. Paulin, and N. M. LeDouarin (1983) Early segregation of a neuronal precursor cell line in the neural crest as revealed by culture in a chemically defined medium. Cell 32:627638. 